\title{
Notch Signaling in Aortic Valve Development and Disease
}

\author{
Vidu Garg
}

\section{Abstract}

Bicuspid aortic valve (BAV) is the most common type of cardiac malformation with an estimated prevalence of $1 \%$ in the population. BAV results in significant morbidity usually during adulthood due to its association with aortic valve calcification and ascending aortic aneurysms. Mutations in the signaling and transcriptional regulator, $\mathrm{NOTCH1}$, are a cause of bicuspid aortic valve in non-syndromic autosomal dominant human pedigrees. The Notch signaling pathway is critical for multiple cellular processes during both development and disease and is expressed in the developing and adult aortic valve consistent with the cardiac phenotypes identified in affected family members. Recent work has begun to elucidate the molecular mechanisms underlying the link between Notch1 signaling and the development of BAV and valve calcification. Using in vitro approaches, loss of Notch signaling has been shown to contribute to aortic valve calcification via Runx2-, Sox9-, and Bmp2-dependent mechanisms. In addition, Notch1 signaling has been shown to be responsive to nitric oxide signaling during this disease process. A new highly penetrant mouse model of aortic valve disease using Notchl haploinsufficient mice that are backcrossed in an endothelial nitric oxide synthase (Nos3)-null background was generated. Notchl and Nos3 compound mutant mice $\left(\mathrm{Notch}^{+/-} ; \mathrm{Nos}^{3^{--}}\right)$display a nearly $100 \%$ incidence of aortic valve malformations, most commonly BAV. The aortic valves of adult mutant mice are thickened and have associated stenosis and regurgitation. Based upon the initial discovery of NOTCHI mutations in

V. Garg, M.D. $(\bowtie)$

Center for Cardiovascular Research and The Heart Center, Nationwide Children's Hospital, 700 Children's Drive Room WB4221, Columbus, OH 43205, USA

Department of Pediatrics (Cardiology), The Ohio State University, Columbus, OH, USA

Department of Molecular Genetics, The Ohio State University, Columbus, OH, USA

e-mail: vidu.garg@nationwidechildrens.org

T. Nakanishi et al. (eds.), Etiology and Morphogenesis of Congenital Heart Disease, DOI 10.1007/978-4-431-54628-3_53 
humans with aortic valve disease, subsequent studies have provided significant molecular insights into BAV-associated diseases.

Keywords

Congenital heart defect • Bicuspid aortic valve • Aortic valve calcification •

Notch signaling

\subsection{Introduction}

Congenital heart disease (CHD) is the most common type of birth defect, with an estimated incidence that ranges from 6 to 19 per 1,000 live births [1]. Even with recent improvements in the care of children, CHD remains a leading cause of infant mortality [2]. The etiology for the majority of cases of CHD remains unknown despite advances in cardiac developmental biology and genetics [3]. While the role of nongenetic causes, such as infectious agents and teratogens, appears to play a causative role in a minority of cases CHD, the role of genetic factors in CHD has become an area of robust investigation. Numerous etiologic genes for CHD have been identified using conventional linkage or candidate gene sequencing approaches and more recently using array-based methodologies or whole exome/ genome sequencing [4].

Within CHD, bicuspid aortic valve (BAV) is the most common congenital cardiac malformation with an estimated prevalence of $1 \%$ in the population. BAV occurs when the aortic valve has only two cusps instead of the normal three [5]. BAV is a common cause of adult valve disease as it is often asymptomatic during childhood. With BAV, the normally thin aortic valve cusps often prematurely calcify leading to valvar thickening and stenosis [5]. BAV may also present with aortic regurgitation and affected individuals are at increased risk for infective endocarditis. BAV is also associated with ascending aortic dilation/aneurysm and may result in the development of aortic dissection [5].

Since early case reports described families with multiple members with BAV nearly four decades ago, several population-based studies have demonstrated a strong genetic component in BAV. This chapter will review how the identification of mutations in NOTCHI in families with inherited BAV have led to an increased understanding of the role of Notch signaling in aortic valve calcification and the generation of novel mouse model of BAV with associated valve disease.

\subsection{NOTCH1 Mutations and Aortic Valve Disease}

We reported a novel genetic etiology of non-syndromic BAV in humans in 2005 [6]. Using a positional cloning approach, a large family with 11 members affected with autosomal dominant aortic valve disease was studied. The primary cardiac malformation in affected family members was BAV. Seven members had 
developed calcification of the aortic valve including four who required surgical valve replacement. The disease locus was mapped to chromosome $9 \mathrm{q} 34$, and subsequent sequencing of a candidate gene, $\mathrm{NOTCH1}$, identified a nonsense mutation in affected family members. In a smaller unrelated family, a NOTCHI frameshift mutation segregated with a similar aortic valve phenotype. Observations of missense NOTCHI mutations in a subset $(\sim 5 \%)$ of individuals with BAV have also been reported with supporting functional data indicating impaired Notch signaling $[7,8]$. These publications suggested that $\mathrm{NOTCHI}$ haploinsufficiency was a cause of BAV in humans.

NOTCHI encodes a single-pass transmembrane receptor and functions in a highly conserved pathway, which plays critical roles in cell fate determination during organogenesis. In mammals, there are four NOTCH receptors (NOTCH14), and they interact with two families of ligands (Jagged 1 and 2 and Delta 1, 3, and 4) [9]. Other Notch family members have been linked to human disease as heterozygous mutations in NOTCH3 have been identified in CADASIL syndrome, while mutations in JAGGED1 and NOTCH2 are found in Alagille syndrome. Targeted disruption of Notchl in mice results in embryonic lethality secondary to vascular defects prior to cardiac valvulogenesis [10]. Each Notch family member has a distinct expression pattern, and Notchl is expressed not only in the endocardium but also the outflow tract cushion mesenchyme during development consistent with the valve phenotype seen in the affected family members [6, 11]. In addition, Notch1 mRNA transcripts are found in the adult murine aortic valve. These findings suggest that Notch1 signaling is important for aortic valve formation and potentially in adult valve diseases.

\subsection{Notch1 Signaling and Aortic Valve Calcification}

With the increased longevity of human population, calcific valvular disease is becoming more prevalent. Calcific aortic stenosis affects an estimated 2-3\% of the population by 65 years of age [12]. Calcification of the normally thin aortic valve cusps leads to valvular thickening with resultant stenosis/regurgitation that ultimately requires surgical replacement. Examination of calcified human valves has demonstrated increased expression of osteogenic markers such as Runx2 [12]. The process of valvular calcification was traditionally proposed to be a degenerative process that occurred with aging, but increasing evidence suggests that molecular pathways underlie this complex disease [12]. In addition to clinical risk factors such as hypertension and hypercholesterolemia, BAV is a major risk factor for CAVD.

The role of the Notch signaling pathway in the development of CAVD has becoming increasingly recognized. Our initial studies demonstrated that Notch1 repressed the activity of Runx2, a transcriptional regulator of osteoblast cell fate [6]. Subsequently our studies focused on the molecular changes that occur with inhibition of Notch signaling in the aortic valve [11]. Consistent with this hypothesis, diseased human aortic valves have decreased expression of NOTCH1 in areas 
of calcium deposition. To identify downstream mediators of Notch1 during valve calcification, the gene expression changes that occur with chemical inhibition of Notch signaling in rat aortic valve interstitial cells (AVICs) were studied. Downregulation of Sox 9 along with several cartilage-specific genes that were direct targets of this transcription factor was identified. Loss of Sox9 has been published to be associated with aortic valve calcification in mouse models [13]. Utilizing an in vitro porcine aortic valve calcification model system, inhibition of Notch activity resulted in accelerated calcification, while stimulation of Notch signaling attenuated the calcific process. Overexpression of Sox 9 was able to prevent the calcification of porcine AVICs that occurs with Notch inhibition. These studies demonstrated that loss of Notch signaling contributes to aortic valve calcification via a Sox9-dependent mechanism. Additional work by other investigators has supported these conclusions and have also demonstrated a role for Bmp2 as a downstream target of Notch1 signaling in this process and found that Notchl haploinsufficient mice develop aortic valve calcification with aging $[14,15]$.

Dysfunction of the valvular endothelium is thought to initiate calcification of neighboring AVICs leading to CAVD. The molecular mechanism by which endothelial cells communicate with AVICs and cause disease is not well understood. Using a coculture and transwell assays, it was shown that a secreted signal from endothelial cells inhibits calcification of porcine AVICs [16]. Nitric oxide (NO), which is secreted by endothelial cells, is critical for numerous physiologic and pathologic processes and had been implicated in the process of aortic valve calcification. In addition, mice lacking Nos3, which encodes for endothelial nitric oxide synthase, display partially penetrant BAV and making $\mathrm{NO}$ a potential candidate for this secreted signal. NO prevents calcification of AVICs in vitro, similar to the presence of endothelial cells, while the absence of NO increases calcification. Overexpression of a constitutively active Notch1 in AVICs prevented calcification that occurs with NO inhibition linking NO and Notch signaling in this process. Consistent with this, endothelial-derived NO signaling increases the expression of a Notch signaling target genes in AVICs and inhibition of NO decreased nuclear localization of NICD in AVICs. Conversely, increased nuclear localization of NICD was noted with the addition of NO donor. Lastly, the NOS3 and Notch1 signaling pathways genetically interact in vivo as NOS3;Notchl compound mutant mice display a highly penetrant aortic valve disease [16]. These mice have highly penetrant BAV and develop hemodynamically significant aortic valve stenosis and regurgitation. These studies suggest that NO signaling in valve endothelial cells regulates Notch1, Sox9, and Bmp2 in the neighboring AVICs and this pathway may be critical in the pathogenesis of adult-onset aortic valve calcification (Fig. 53.1).

\subsection{Future Directions and Clinical Implications}

While mutations in NOTCHI were identified in a family with a common cardiac valve malformation, subsequent work has demonstrated a role for Notch1 in aortic valve calcification. The development of a highly penetrant mouse model of BAV 


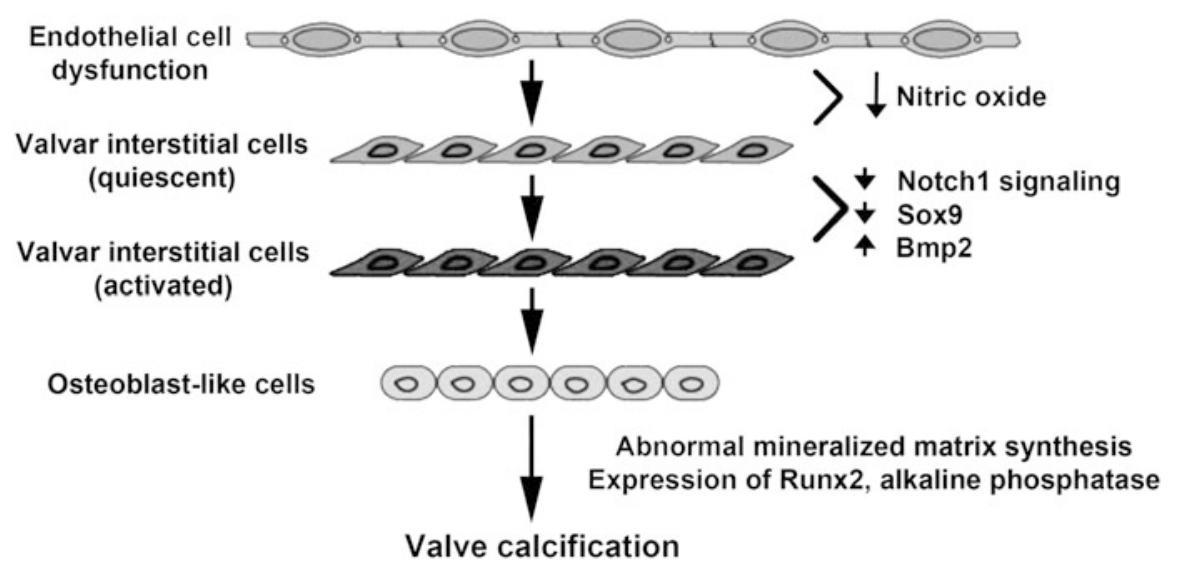

Fig. 53.1 Role of Notch1 signaling in aortic valve calcification. Endothelial cell dysfunction results in decreased nitric oxide production which decreases Notch1 signaling in aortic valve interstitial cells and leads to calcification by Sox9-, Bmp2-, and Runx2-dependent mechanisms

will assist in the dissection of the molecular pathways that lead to the development of this common cardiac malformation. Interestingly, mice deficient for Gata5 display partially penetrant BAV and have reduced expression of Nos3 and Notch signaling [17]. These mice also offer an opportunity to study the development of $\mathrm{BAV}$-associated ascending aortic aneurysms. Future investigations into the downstream targets of Notch1 signaling may lead to novel therapies for BAV-associated diseases such as CAVD.

Acknowledgments V.G. is supported by funding from NIH/NHLBI and Nationwide Children's Hospital.

Open Access This chapter is distributed under the terms of the Creative Commons AttributionNoncommercial 2.5 License (http://creativecommons.org/licenses/by-nc/2.5/) which permits any noncommercial use, distribution, and reproduction in any medium, provided the original author(s) and source are credited.

The images or other third party material in this chapter are included in the work's Creative Commons license, unless indicated otherwise in the credit line; if such material is not included in the work's Creative Commons license and the respective action is not permitted by statutory regulation, users will need to obtain permission from the license holder to duplicate, adapt or reproduce the material.

\section{References}

1. Hoffman JI, Kaplan S. The incidence of congenital heart disease. J Am Coll Cardiol. 2002;39:1890-900.

2. Go AS, Mozaffarian D, Roger VL, et al. Heart disease and stroke statistics - 2013 update: a report from the American Heart Association. Circulation. 2013;127:e6-245. 
3. Fahed AC, Gelb BD, Seidman JG, et al. Genetics of congenital heart disease: the glass half empty. Circ Res. 2013;112:707-20.

4. Pierpont ME, Basson CT, Benson Jr DW, et al. Genetic basis for congenital heart defects: current knowledge: a scientific statement from the American Heart Association Congenital Cardiac Defects Committee, Council on Cardiovascular Disease in the Young: endorsed by the American Academy of Pediatrics. Circulation. 2007;115:3015-38.

5. Ward C. Clinical significance of the bicuspid aortic valve. Heart. 2000;83:81-5.

6. Garg V, Muth AN, Ransom JF, et al. Mutations in NOTCH1 cause aortic valve disease. Nature. 2005;437:270-4.

7. McBride KL, Riley MF, Zender GA, et al. NOTCH1 mutations in individuals with left ventricular outflow tract malformations reduce ligand-induced signaling. Hum Mol Genet. 2008;17:2886-93.

8. McKellar SH, Tester DJ, Yagubyan M, et al. Novel NOTCH1 mutations in patients with bicuspid aortic valve disease and thoracic aortic aneurysms. J Thorac Cardiovasc Surg. 2007;134:290-6.

9. de la Pompa JL, Epstein JA. Coordinating tissue interactions: notch signaling in cardiac development and disease. Dev Cell. 2012;22:244-54.

10. Krebs LT, Xue Y, Norton CR, et al. Notch signaling is essential for vascular morphogenesis in mice. Genes Dev. 2000;14:1343-52.

11. Acharya A, Hans CP, Koenig SN, et al. Inhibitory role of Notch1 in calcific aortic valve disease. PLoS One. 2011;6:e27743.

12. Garg V. Molecular genetics of aortic valve disease. Curr Opin Cardiol. 2006;21:180-4.

13. Peacock J, Levay AK, Killaspie DB, et al. Reduced sox 9 function promotes heart valve calcification phenotypes in vivo. Circ Res. 2010;106(4):712-9.

14. Nigam V, Srivastava D. Notch1 represses osteogenic pathways in aortic valve cells. J Mol Cell Cardiol. 2009;47:828-34.

15. Nus M, MacGrogan D, Martinez-Poveda B, et al. Diet-induced aortic valve disease in mice haploinsufficient for the Notch pathway effector RBPJK/CSL. Arterioscler Thromb Vasc Biol. 2011;31(7):1580-8.

16. Bosse K, Hans CP, Zhao N, et al. Endothelial nitric oxide signaling regulates Notch1 in aortic valve disease. J Mol Cell Cardiol. 2013;60:27-35.

17. Laforest B, Andelfinger G, Nemer M. Loss of Gata5 in mice leads to bicuspid aortic valve. J Clin Invest. 2011;121:2876-87. 